\section{Malignant Struma Ovarii Requiring Emergency Surgery for Acute Abdomen: A Case Report and Literature Review}

\section{Abstract}

Malignant struma ovarii is an extremely rare tumor, accounting for $\leq 0.1 \%$ of all mature cystic teratomas and $0.01 \%$ of all ovarian tumors. We herein describe a case involving a patient who underwent emergency surgery for suspected torsion of a mature cystic teratoma that was later diagnosed as malignant struma ovarii. A 23-year-old woman was brought to our hospital with the main complaint of acute lower abdominal pain. Computed tomography revealed a $10-\mathrm{cm}$-diameter right ovarian tumor with findings suggestive of a mature cystic teratoma. Based on the computed tomography and clinical findings, torsion of the ovarian tumor was suspected; therefore, emergency laparoscopic-assisted surgery was performed. On histopathological examination, the tumor was composed of cells with clear basophilic cytoplasm and a round nucleus. The cells were organized in thyroid follicle-like structures or funicular and solid honeycomb structures, and they exhibited slight nuclear atypia, proliferation, and vascular invasion. The tumor was diagnosed as malignant struma ovarii, and additional surgical resection of the right adnexa with partial omentectomy by laparotomy was performed on a later date. No malignant cells were found in the subsequently resected tissues, and cytologic examination of the ascitic fluid was negative. Because no residual tumor was found, the patient was followed up at the outpatient clinic without additional treatment. No signs of recurrence had been detected at the time of this writing. We herein present this case along with a literature review.

Keywords: Malignant struma ovarii; Emergency operation; Diagnosis; Preservation of fertility

\section{Isao Murakami", Kyoko Tanaka ${ }^{1 *}$ and Daisuke Aoki²}

1 Department of Gynecology, Toho University Ohashi Medical Center, Tokyo, Japan

2 Department of Obstetrics and Gynecology, Keio University School of Medicine, Tokyo, Japan

*Corresponding author: Kyoko Tanaka

ほ kyoko.tanaka@med.toho-u.ac.jp

Department of Gynecology, Toho University Ohashi Medical Center 2-22-36 Oohashi, Meguro-ku, Tokyo 153-8515, Japan.

Tel: +81-3-3468-1251

Citation: Murakami I Tanaka K, Aoki D (2020) Malignant Struma Ovarii Requiring Emergency Surgery for Acute Abdomen: A Case Report and Literature Review. Gynecol Obstet Case Rep Vol.6 No.1:5

\section{Introduction}

Malignant struma ovarii is an ovarian tumor histologically composed of thyroid tissue that occupies $\geq 50 \%$ of the tumor, with nuclear atypia of the constituent cells and evidence of vascular invasion. It is a very rare ovarian tumor, accounting for $\leq 0.1 \%$ of all mature cystic teratomas and $0.01 \%$ of all ovarian tumors. Most signs and symptoms of this tumor are nonspecific and include lower abdominal pain and a lower abdominal mass. Thus, struma ovarii is often found incidentally during surgery or as a cause of acute abdomen. Sufficient evidence regarding the optimal treatment of malignant struma ovarii has not been obtained because of the scarcity of cases. In particular, only a few reports have addressed the treatment policy that must be adopted to preserve fertility while reducing the risk of recurrence in patients with this tumor. Given this paucity of data, the impact of malignant struma ovarii on life expectancy remains controversial, and its optimal management is currently debated [1-4]. Most authors advocate for aggressive treatment involving local surgery followed by total thyroidectomy, radioactive iodine ablation, and thyroid hormone-suppressive therapy regardless of the presence of distant metastases at the time of diagnosis [5-9]. We encountered a patient who underwent emergency surgery (laparotomic tumorectomy) for suspected torsion of an ovarian tumor that was later histopathologically diagnosed as malignant struma ovarii. We herein present this case along with a literature review. 


\section{Case Report}

A 23-year-old woman (nullipara, nulligravida) was brought to our hospital by ambulance because of acute lower abdominal pain that had developed several days previously. Although she had been diagnosed with an ovarian tumor by a local doctor, she had received no specific treatment. She had no remarkable medical or family history.

On admission, pelvic examination revealed an increase in the size of the right adnexa, with marked tenderness on pressure. No abnormalities were found in the uterus or left adnexa. Ultrasonography showed a right ovarian mass measuring $10 \mathrm{~cm}$ in diameter. Laboratory examinations, including blood counts and biochemical tests, revealed no significant abnormalities with the exception of an elevated serum C-reactive protein concentration of $8.71 \mathrm{mg} / \mathrm{dl}$. Urgent contrast-enhanced computed tomography showed a tumor measuring $10 \mathrm{~cm}$ in diameter in the right ovary. The tumor consisted of a cystic component containing fatty material coexisting with a solid component, and it showed strong contrast enhancement suggestive of bleeding. Based on these findings, torsion of a mature cystic teratoma was suspected (Figure 1), and emergency laparoscopic-assisted tumorectomy was carried out.

During surgery, a 10-cm-diameter right ovarian tumor with a smooth surface was observed; however, there no torsion, adhesion, or ascites was present. A well-developed atypical vessel inside the tumor was damaged during the surgery, resulting in heavy bleeding. Therefore, the surgical procedure was converted to laparotomy and the tumor was resected. The intraoperative blood loss volume was $640 \mathrm{ml}$. The tumor contained some fatty material and hair, but for the most part, it was solid with some atypical vessels (Figure 2). The patient's postoperative course was favorable, and she was discharged 7 days after the surgery.

On histopathological examination, the resected tumor was lined by epidermoid stratified squamous epithelium and contained glial and cartilage tissues in the cystic part. The solid part was mostly composed of proliferative cells with clear basophilic cytoplasm that were organized into thyroid follicle-like structures (Figure 3 ). In some areas, the cells formed funicular and solid honeycomb structures. The cells were relatively uniform in size and shape, but they showed an increased nuclear/cytoplasmic ratio; nuclear atypia and evidence of vascular invasion were also found (Figure 4). Immunohistochemical tests showed positive results for anti-thyroid transcription factor 1 antibody and antithyroglobulin antibody, which are thyroid follicle epithelium markers (Figure 5). Based on these histopathological findings, the tumor was diagnosed as malignant struma ovarii containing a poorly differentiated carcinoma component.

Considering the patient's age, preservation of fertility was attempted. Resection of the adnexa only on the affected side and partial omentectomy were performed at a later date as an additional surgery. At the time of the additional surgery, cytologic examination of the peritoneal washings showed negative results, and the resected tissues showed no evidence of malignancy. The patient showed a favorable postoperative

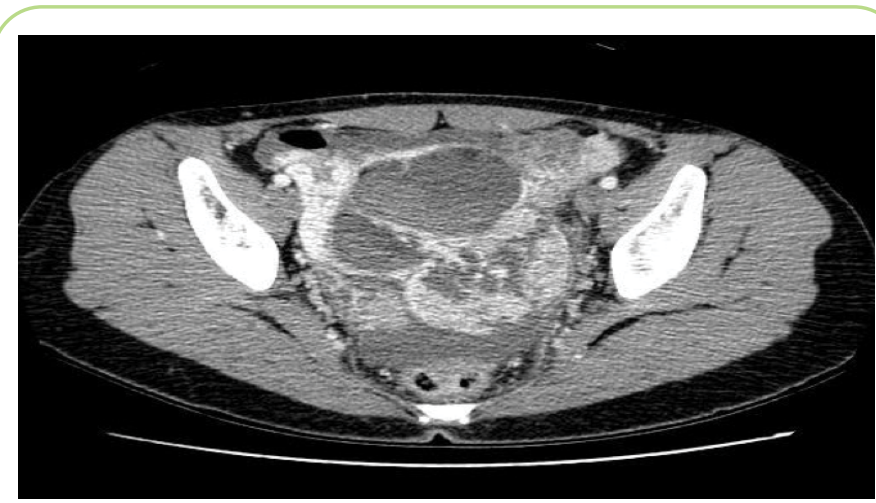

Figure 1 Transverse computed tomography section. The section shows a $10-\mathrm{cm}$ well-defined complex lesion with solid and cystic components in the pelvis, extending on either side of the midline and reaching to both sides of the adnexa. No obvious lymph nodes or signs of metastasis are present.

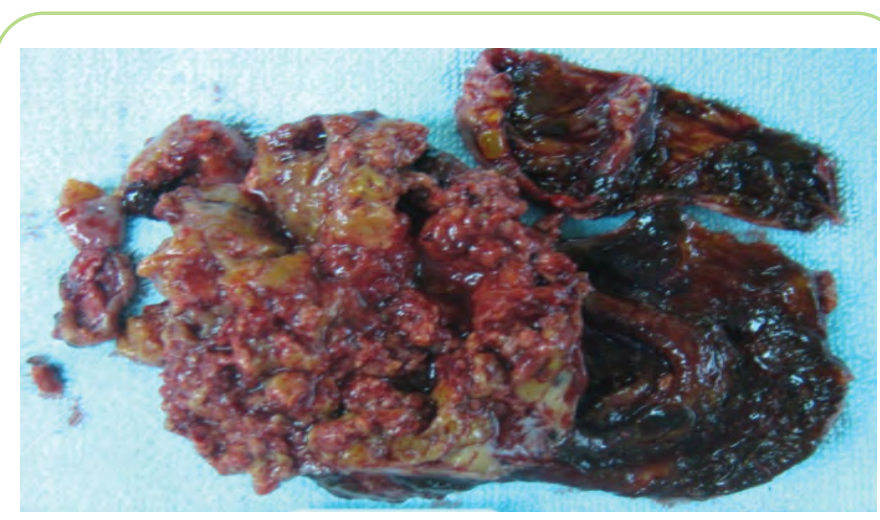

Figure 2 Macroscopic findings. A 7-cm nodule of malignant struma ovarii in the wall of a $10-\mathrm{cm}$ dermoid cyst.

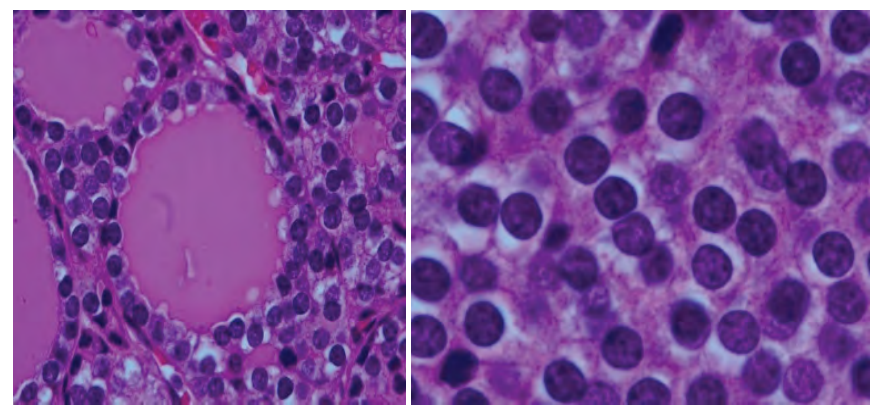

Figure 3 Low-power (200x) and high-power (400x) hematoxylinand eosin-stained sections. (A) Low-power hematoxylinand eosin-stained section demonstrates a thyroid follicle pattern. (B) High-power hematoxylin- and eosin-stained section demonstrates nuclear features including nuclear grooves, clear overlapping, and enlargement.

course and was discharged 7 days postoperatively. We explained to the patient and her family that she had a very rare tumor for which there was no established treatment and that a favorable prognosis could be expectable with 1131 radiation therapy even if the disease recurred. In deference to the wishes of the patient 


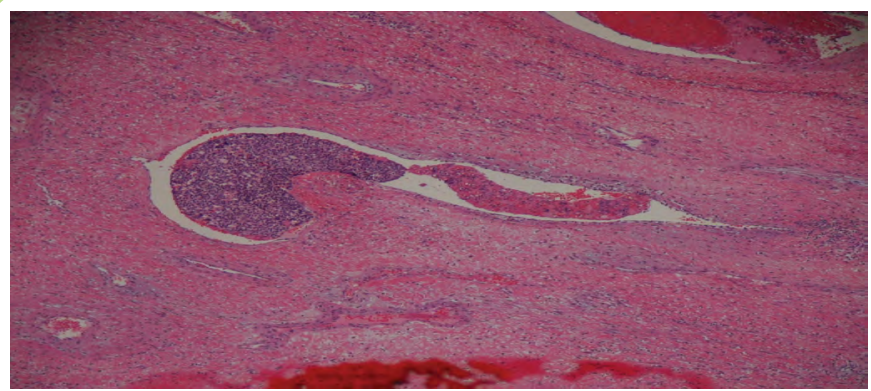

Figure 4 Vascular invasion in follicular adenocarcinoma (100x).

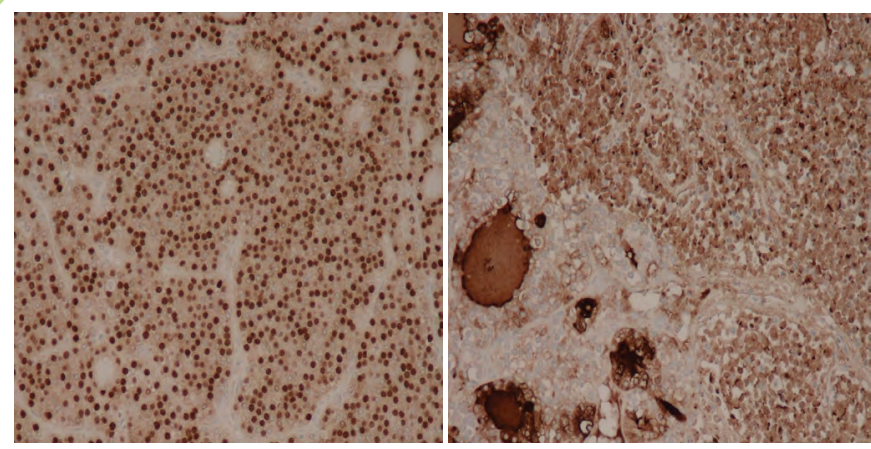

Figure 5 Immunohistochemical sections. Immunohistochemical tests showed positive results for (A) anti-thyroid transcription factor 1 antibody (250x) and (B) antithyroglobulin antibody (250x).

and her family, the patient was followed at our outpatient clinic without radiation therapy because of concerns regarding the risk of infertility with such therapy. At the time of this writing (12 months after the initial emergency surgery), the patient was still being followed up and had shown no signs of tumor recurrence.

\section{Discussion}

Malignant struma ovarii is an ovarian germ cell tumor classified as a teratoma. It is a very rare tumor, accounting for $\leq 0.1 \%$ of all mature cystic teratomas and $0.01 \%$ of all ovarian tumors [9-13]. The diagnostic criteria are in accordance with those of thyroid cancer. Malignant struma ovarii is defined as an ovarian tumor histologically composed of thyroid tissue accounting for $\geq 50 \%$ of the solid part of the tumor and showing evidence of malignancy, such as vascular invasion. Morphologically, this tumor contains thyroid follicle-like structures and papillary structures and may exhibit proliferation of undifferentiated cells. To confirm the presence of thyroid tissue, immunohistochemical assays with anti-triiodothyronine, anti-thyroxine, anti-thyroid transcription factor 1, and anti-thyroglobulin antibodies are useful. Findings suggestive of malignancy include distant metastasis, nuclear atypia, nuclear enlargement, and vascular invasion. In particular, papillary cancer is characterized by the presence of "ground glass" nuclei [9,12-14]. Pathological examination in our case showed relatively uniform-looking tumor cells with slight nuclear atypia, evidence of vascular invasion, and proliferation of poorly differentiated cells. A diagnosis of malignant struma ovarii was made based on these findings (Figures $\mathbf{3}$ and 5).
The clinical manifestations of malignant struma ovarii are nonspecific and include abdominal pain, an abdominal mass, irregular genital bleeding, and ascites. This disease is often found incidentally during abdominal surgery or imaging studies. Differentiation from a teratoma is difficult by diagnostic imaging. Blood thyroid hormone levels are usually within normal limits, and symptoms of hyperthyroidism are relatively rare, occurring in only $5 \%$ to $8 \%$ of patients with this disease $[9,12]$. However, elevated blood thyroglobulin levels have been reported; therefore, blood thyroglobulin has been used as a tumor marker for malignant struma ovarii $[9,12]$.

Because of the limited number of patients with malignant struma ovarii seen in routine clinical practice, no standard treatment has been established. Malignant struma ovarii is classified as a germ cell tumor in the General Rules for Clinical Management of Ovarian Tumours, and surgical treatment followed by chemotherapy is recommended by the NCCN Guidelines Version 4,2017 . To the best of our knowledge, however, the only report of postoperative chemotherapy following surgical treatment was by Robboy et al. [14] in 2009 that used phenylalanine mustard, adriamycin, and vincristine. Thus, the efficacy of chemotherapy for malignant struma ovarii remains unclear. Several patients have reportedly undergone the same surgical treatment as for ovarian cancer (hysterectomy, bilateral adnexectomy, and retroperitoneal node dissection) with subsequent $\mathbf{1 3 1}$ radiation therapy. Thyroidectomy should precede $I 131$ radiation therapy to allow adequate accumulation of the radionuclide in the lesion. Following surgical treatment combined with 1131 radiation therapy, the 10 -year survival rate is reportedly $89 \%$ and the 25 year survival rate is $84 \%$ [15]. Research has also shown that the recurrence rate after treatment is $38 \%$ among all patients with malignant struma ovarii and that the recurrence rate after initial surgical treatment not followed by radiation therapy is 50\% [9].

Adnexectomy only on the affected side and omentectomy are performed when preservation of fertility is desired [9-11]. Although the influence of $\mathrm{I} 131$ radiation therapy itself on infertility is regarded as minimal, radiation therapy following surgical resection is often avoided in patients desiring preservation of fertility, especially considering the potential need for thyroid hormone replacement after thyroidectomy. However, when only adnexectomy on the affected side is performed for fertility preservation, subsequent recurrence is relatively frequent. Among 24 patients with malignant struma ovarii reported from 1970 to 2003, 9 patients underwent adnexectomy only on the affected side. Three of these nine patients received $I 131$ radiation therapy immediately after the surgery, and none developed recurrence $[9,11,15-18]$. The remaining six patients were followed up without radiation therapy after the adnexectomy, and five of these patients developed recurrence (Table 1). There were variations in the lesion stage at the time of the initial surgery, and the time to recurrence varied from 4 to 108 months. The mode of recurrence was peritoneal dissemination in four of the five patients. No patients died of recurrence because all patients with recurrence responded to treatment, including I131 radiation therapy. The longest reported survival period is 432 
Table 1 Summary of cases of malignant struma ovarii without radiation therapy.

\begin{tabular}{|c|c|c|c|c|}
\hline Patient No. & Initial therapy & Additional treatment & Recurrence (months) Therapy for recurrence & Overall survival (months) \\
\hline 1 & LSO & None & None & 36 \\
\hline 2 & LSO & None & 4 TAH+RSO+Thyroidectomy+1 ${ }^{131}$ & 15 \\
\hline 3 & RSO & None & 8 Thyroidectomy+1 I $^{131}$ & 29 \\
\hline 4 & RSO & None & $24 \mathrm{TAH}+$ LSO+Thyroidectomy+1 I $^{131}$ & 62 \\
\hline 5 & RSO & None & $14 \mathrm{TAH}+$ LSO+Thyroidectomy+1 & 432 \\
\hline
\end{tabular}

months; thus, a relatively good prognosis can be expected with appropriate treatment [11,14,16-18].

Risk factors for recurrence and metastasis include adhesions observed during surgery, an ascitic fluid volume of $>1000 \mathrm{ml}$, destruction of the ovarian serosa, the presence of papillary tissue, and a tumor diameter of $\geq 12 \mathrm{~cm}$ [9]. Our patient has a substantial risk of recurrence in light of these factors and therefore requires close follow-up observation.

\section{Conclusion}

In conclusion, patients with malignant struma ovarii may exhibit clinical signs and symptoms and imaging findings similar to those in patients with a benign ovarian tumor. Careful clinical attention is needed to distinguish between mature cystic teratoma and

\section{References}

1 Krishnamurthy A, Ramshankar V, Vaidyalingam V, Majhi U (2013) Synchronous papillary carcinoma thyroid with malignant struma ovarii: A management dilemma. Indian J Nucl Med 28: 243-245.

2 Marti JL, Clark VE, Harper H, Chhieng DC, Sosa JA, et al. (2012) Optimal surgical management of well-differentiated thyroid cancer arising in struma ovarii: A series of 4 patients and a review of 53 reported cases. Thyroid 22: 400-406.

3 Mattucci ML, Dellera A, Guerriero A, Barbieri F, Minnelli L, et al. (2007) Malignant struma ovarii: a case report and review of the literature. J Endocrinol Invest 30: 517-520.

4 Sussman SK, Kho SA, Cersosimo E, Heimann A (2002) Co-existence of malignant struma ovarii and Graves' disease. Endocr Pract 8: 378-380.

5 Shrimali RK, Shaikh G, Reed NS (2012) Malignant struma ovarii: The west of Scotland experience and review of literature with focus on postoperative management. J Med Imaging Radiat Oncol 56: 478-482.

6 Leong A, Roche PJ, Paliouras M, Rochon L, Trifiro M, et al. (2013) Coexistence of malignant struma ovarii and cervical papillary thyroid carcinoma. J Clin Endocrinol Metab 98: 4599-4605.

7 Matysiak-Grzes M, Fischbach J, Gut P, Klimowicz A, Gryczynska M, et al. (2013) Struma ovarii maligna. Neuro Endocrinol 34: 97-101.

8 Yoo SC, Chang KH, Lyu MO, Chang SJ, Ryu HS, et al. (2008) Clinical characteristics of struma ovarii. J Gynecol Oncol 19: 135-138.

9 DeSimone CP, Lele SM, Modesitt SC (2003) Malignant struma ovarii: rare tumors such as malignant struma ovarii. Efforts should be pursued to standardize the care of patients with malignant struma ovarii, perhaps with practice guidelines. We hope that population-level data such as these will be the first step in this process.

\section{Acknowledgement}

We thank Angela Morben, DVM, ELS, from Edanz Group (https:// en-author-services.edanzgroup.com/), for editing a draft of this manuscript.

\section{Conflict of Interest}

No potential conflict of interest relevant of this article was reported.

A case report and analysis of cases reported in the literature with focus on survival and I131 therapy. Gynecol Oncol 89: 543-548.

10 Zakhem A, Aftimos G, Kreidy R, Salem P (1990) Malignant struma ovarii: A report of two cases and selected review of the literature. $J$ Surg Oncol 43: 61-65.

11 Dardik RB, Dardik M, Westra W, Fredrick M (1999) Malignant struma ovarii: Two case reports and a review of the literature. Gynecol Oncol 73: $447-451$

12 Yoo SC, Chang KH, Lyu MO, Chang SJ, Ryu HS, et al. (2008) Clinical characteristics of struma ovarii. J Gynecol Oncol 19: 135-138.

13 Devaney K, Snyder R, Norris H, Tavassoli FA (1993) Proliferative and histologically malignant struma ovarii: a clinicopathologic study of 54 cases. Int J Gynecol Pathol 12: 333-343.

14 Robboy SJ, Shaco-Levy R, Peng RY, Snyder MJ, Donahue J, et al. (2009) Malignant struma ovarii: an analysis of 88 cases, including 27 with extraovarian spread. Int J Gynecol Pathol 28: 405-422.

15 Roth LM, Talerman A (2007) The enigma of struma ovarii. Pathology 39: 139-146.

16 Willemse PHB, Oosterhuis JW, Aalders JG, Piers DA, Sleijfer DT, et al. (1987) Malignant struma ovarii treated by ovariectomy, thyroidectomy, and I-131 administration. Cancer 60: 178-182.

17 Rosenblum N, LiVolsi V, Edmonds P, Mikuta J (1989) Malignant struma ovarii. Gynecol Oncol 32: 575-577.

18 O'Connell M, Fisher C, Harmer C (1989) Malignant struma ovarii: Presentation and management. Br J Radiol 63: 360-362. 